\title{
NUMERICAL ANALYSIS OF BURIED VIBRATION PROTECTION DEVICES USING THE METHOD OF FUNDAMENTAL SOLUTIONS
}

\author{
CARLOS ALBINO $^{1}$, LUÍS GODINHO ${ }^{1}$, DANIEL DIAS-DA-COSTA ${ }^{1,2}$ \& PAULO AMADO-MENDES \\ ${ }^{1}$ ISISE, Department of Civil Engineering, University of Coimbra, Portugal \\ ${ }^{2}$ School of Civil Engineering, The University of Sydney, Australia
}

\begin{abstract}
Buried structures may be used to control elastic wave propagation in soils and help to reduce vibrations in sensitive structures. The analysis of these structures using numerical tools is of high importance and is usually a demanding computational task. In the present work, the authors explore the possibility of using a meshless method for such simulations, namely the method of fundamental solutions (MFS). In many applications, the MFS has proved to be a worthy and more efficient alternative to classic methods, such as the FEM or even the BEM. Here, the authors present a study on the application of the MFS to simulate the propagation of elastic waves in a soil with multiple buried inclusions. Application examples are also presented, in which the vibration reduction provided by different sets of buried inclusions is analysed and discussed.
\end{abstract}

Keywords: elastodynamics, MFS, numerical modelling, periodic structures, vibration filtering.

\section{INTRODUCTION}

Vibration induced in soils by dynamic sources, particularly transportation means, can affect sensitive constructions, and can be nowadays a strong limitation to the implementation of new infrastructures, such as railways. Mitigation of vibrations affecting sensitive constructions has been discussed since the middle of last century when high-speed trains with speeds above $200 \mathrm{~km} / \mathrm{h}$ - emerged as regular intercity transport [1]. Nowadays, with the railways crossing urban areas and with an increase in the quality of life, people increasingly complain about the noise and vibrations caused by the passage of trains [2], with the noise and vibration exposure leading to an increased number of awakenings and even cardiac problems [3]. These vibrations induced by the activity of rail transport systems can be mitigated by reducing vibration at source (train, train-track interaction, railway sleepers, etc.) or at a distance from this source, intervening in the host medium, reducing the propagation of waves (trenches, buried structures, etc.). Different authors studied how to reduce the vibration emitted by the source [4] and investigated the mitigation of the vibrations through structures constructed in the host medium [5]. However, simulating these structures requires demanding computational resources if traditional numerical techniques, such as the Finite Element Method (FEM) or the Boundary Element Method (BEM), are to be used. For the FEM, the large finite element mesh required to simulate wave propagation problems can be a significant limitation for its use. The BEM [6], [7] seems to be a very interesting alternative to the FEM, since it allows a very compact description of the propagation medium, requiring only discretization of the interfaces between different materials, and so it has indeed received great interest from the scientific community [8], [9]. However, the BEM also exhibits some limitations, since it produces fully filled equation systems, requires the previous knowledge of the fundamental solutions for the physical problem, and exhibits some additional mathematical complexity due to the need of calculating singular or quasi-singular integrals.

In the last two decades meshless methods emerged trying to overcome some of the difficulties of the conventional methods. Among other advantages, in many cases those 
methods allow reducing the computational cost [10], and can be quite simple to implement. An interesting and accepted approach that found applications in many domains is the method of fundamental solutions (MFS). In the case of wave propagation problems, the MFS displacement field is simulated by the superposition of fields generated by a number of virtual sources located outside the domain. The errors of the method can be controlled and minimized through the right choice of the virtual sources and collocation points used to define the solution and enforce the boundary conditions, although this is still an active research topic [11], [12]. In a recent work [13], the singular boundary method (SBM) was also proposed, based on the method of fundamental solutions (MFS). In the SBM, unlike the MFS, virtual sources coinciding with collocation points in the physical boundary of the problem are used to simulate the displacement field and an inverse interpolation technique regularises the singularity of the fundamental solution. In an attempt to combine the advantages of different methods, some researchers have also developed strategies to combine and couple different methods for some specific problems (see for example [14], [15]).

In the present work, the authors make use of the MFS to simulate the propagation of waves in the presence of a set of periodically spaced elastic inclusions buried in a half-space. The main goal is to better understand the possibility of using such periodic systems for the mitigation of propagating vibrations induced by different sources. In what follows, first, a brief description of the governing equations is given, followed by an overview of the MFS formulation and of the involved Green's functions. Then, a study on the accuracy of the method is given. Finally, some numerical examples are presented to illustrate the main results computed in the scope of this work.

\section{MATHEMATICAL FORMULATION}

\subsection{Wave propagation}

In the time domain, wave propagation in a homogeneous elastic medium follows the equilibrium equation [9]:

$$
\sigma_{i j, j}=\rho \frac{\partial^{2} \widehat{u}_{i}}{\partial t^{2}},
$$

where $\sigma_{i j}, \hat{u}_{i}$ and $\rho$ are the stress tensor, the mechanical displacements and the volumetric mass density of the elastic medium, respectively, and the indices $i$ and $j$ can denote either $x$ or $y$. The comma followed by the index denotes the partial differentiation with respect to the spatial coordinate $x_{i}$. The elastic stress tensor is defined as

$$
\sigma_{i j}=\lambda \delta_{i j} u_{k, k}+\mu\left(u_{i, j}+u_{j, i}\right),
$$

where $\lambda$ and $\mu$ are the Lamé material constants, and $\delta_{i j}$ is the Kronecker delta function. Following eqns (1) and (2), it can be written that

$$
(\lambda+2 \mu) \nabla(\nabla \cdot \widehat{\mathbf{u}})-\mu \nabla \times(\nabla \times \widehat{\mathbf{u}})-\rho \frac{\partial^{2} \widehat{\mathbf{u}}}{\partial t^{2}}=0,
$$

where $\widehat{\mathbf{u}}$ is the displacement vector. The application of a time Fourier transformation leads to the following equation, when null initial conditions are assumed:

$$
(\lambda+2 \mu) \nabla \nabla \cdot \mathbf{u}-\mu \nabla \times \nabla \times \mathbf{u}+\omega^{2} \rho \mathbf{u}=0,
$$

in which $\omega$ is the angular frequency and $\mathbf{u}$ is the displacement vector in the frequency domain. Here, the particular case of a plane strain problem is addressed. The vector equation can be 
solved using the MFS which approximates the solution within a given elastic domain as a combination of fundamental solutions related to a set of virtual sources positioned outside the domain to avoid singularities.

\subsection{General formulation of the MFS}

Consider a solid inclusion $\Omega_{i}$, with density $\rho_{i}$ - where compressional waves (P) and shear waves (S) propagate with $\alpha_{i}$ and $\beta_{i}$ velocities, respectively, embedded in an elastic host medium $\Omega_{h}$, with density $\rho_{h}$ which allows $\mathrm{P}$ and $\mathrm{S}$ waves to propagate with $\alpha_{h}$ and $\beta_{h}$ velocities. The host medium is excited by a dynamic load $F$ at position $P_{0}\left(x_{0}, y_{0}\right)$ oscillating with a frequency $\omega$, according to the Fig. 1 .

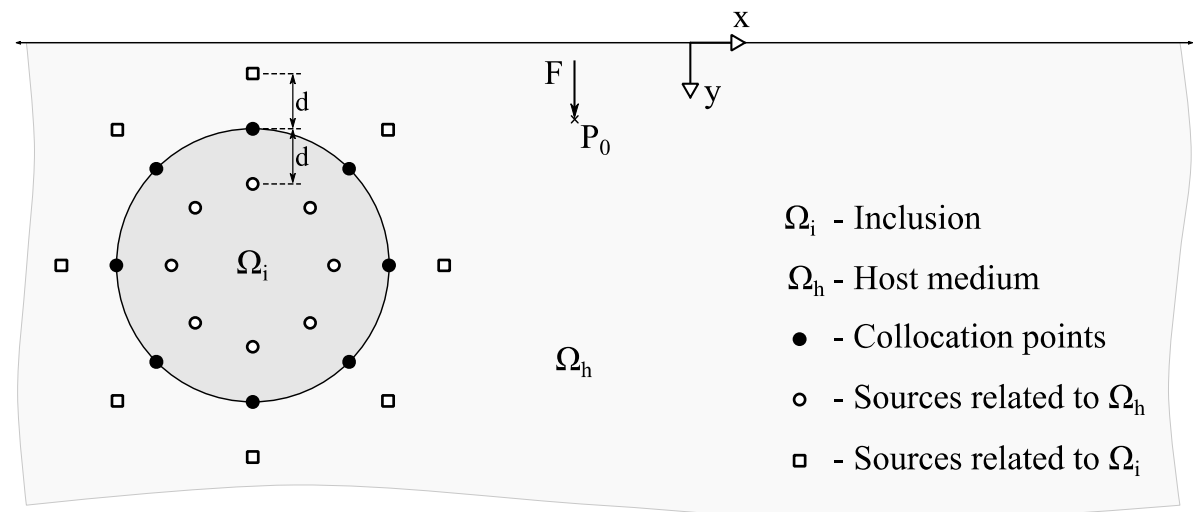

Figure 1: Schematic representation of MFS model.

The wave field can be approximated using the MFS by linearly superimposing the effects of a number of virtual sources located outside the propagation domain [12], [13]. In the present case, two sets of $N C$ virtual sources placed on either side of that interface at a distance $d$ are considered. At each position, virtual loads must be considered acting in both directions $x$ and $y$. Sources inside the inclusion (related to the host medium) have unknown amplitudes $P_{n j}$ whereas those placed in the host medium (related to the inclusion) have unknown amplitudes $Q_{n j} ; n$ is the source order number and $j$ is the direction in which the load acts on this source. Accordingly, in the host medium, the displacements are given by:

$$
u_{i}^{h}(x, y)=\sum_{n=1}^{N C} \sum_{j=1}^{2} P_{n j} G_{i j}^{h}\left(x_{n}^{h}, y_{n}^{h}, x, y, \rho_{h}, \alpha_{h}, \beta_{h}\right),
$$

while inside the inclusion:

$$
u_{i}^{m}(x, y)=\sum_{n=1}^{N C} \sum_{j=1}^{2} Q_{n j} G_{i j}^{m}\left(x_{n}^{m}, y_{n}^{m}, x, y, \rho_{m}, \alpha_{m}, \beta_{m}\right),
$$

where $G_{i j}^{m}(\cdot)$ is the displacement at point $(x, y)$, in medium $m$, along direction $i$, generated by a load acting along $j$ at position $\left(x_{n}^{m}, y_{n}^{m}\right)$, computed using the adequate Green's functions [16], [17]. 
To determine the unknown amplitudes, it is necessary to impose the boundary conditions of continuity of tangential and normal displacements and stresses along the interface, at $N C$ collocation points, establishing a linear equation system with $4 N C$ equations and $4 N C$ unknowns. After solving these equations, the displacements at any point $(x, y)$ in the propagation domain can be determined as a summation of the contribution of their respective virtual sources.

In the analysed scenarios, the system of equations is generated from two sets of fundamental solutions: one corresponding to the Green's functions for an elastic half-space simulating the host medium and another being the Green's functions for an elastic full-space simulating the field within the inclusion.

\subsection{Green's functions}

Both sets of fundamental solutions, which relate the field variables (stresses or displacements) at some location in the domain caused by a dynamic source placed elsewhere in the medium, are extensively described in works by Tadeu et al. [16] and Tadeu and Kausel [17]. To avoid the discretization of the horizontal surface of the half-space, Green's functions that take its presence into account are used, following the works of these latter authors. For a half-space medium, the total wave field can be expressed by taking into account the incident field generated by the source (source terms), and the terms generated at the surface (surface terms). The source terms can be written making use of the equations proposed by Tadeu and Kausel [17] for 2D loads, while surface terms can be represented by a set of one dilatational, $\phi$, and another shear, $\psi$, potentials with unknown amplitude values. The continuous integrals of the effects of plane waves which express both the source and the surface terms, can be discretized into summations of discrete terms, assuming the existence of an infinite number of virtual sources placed along the $x$ direction at equal intervals, $L_{x}$. The distance separating them is large enough to prevent the virtual loads from contaminating the response. Considering the contribution of $2 N+1$ terms, the potentials that define the surface terms for loads acting along the $x$ direction are

$$
\phi_{0}^{x}=E_{a} \sum_{n=-N}^{n=+N}\left(\frac{k_{n}}{v_{n}} E_{b 0} A_{n}^{x}\right) E_{d} ; \psi_{0}^{x}=-E_{a} \sum_{n=-N}^{n=+N}\left(E_{c 0} C_{n}^{x}\right) E_{d},
$$

and for loads acting along the $y$ direction

$$
\phi_{0}^{y}=E_{a} \sum_{n=-N}^{n=+N}\left(E_{b 0} A_{n}^{y}\right) E_{d} ; \psi_{0}^{y}=E_{a} \sum_{n=-N}^{n=+N}\left(\frac{k_{n}}{\gamma_{n}} E_{c 0} B_{n}^{y}\right) E_{d},
$$

where $A_{n}^{x}, A_{n}^{y}, B_{n}^{y}$ and $C_{n}^{x}$, are the unknown coefficient to be determined from appropriate boundary conditions: $E_{a}=\frac{1}{2 \rho_{1} \omega^{2} L_{x}} ; E_{b 0}=e^{-i v_{n} y} ; E_{c 0}=e^{-i \gamma_{n} y} ; E_{d}=e^{-i k_{n}\left(x-x_{0}\right)} ; i=$ $\sqrt{-1} ; k_{n}=2 \pi n / L_{x}$ (the horizontal wavenumber along $x$ ); $k_{p 1}=\omega / \alpha_{1} ; k_{s 1}=\omega / \beta_{1} ; \gamma_{n}=$ $\sqrt{k_{s 1}^{2}-k_{n}^{2}}\left(\right.$ where $\left.\operatorname{Im}\left(\gamma_{n}\right) \leq 0\right) ; v_{n}=\sqrt{k_{p 1}^{2}-k_{n}^{2}}\left(\right.$ where $\left.\operatorname{Im}\left(v_{n}\right) \leq 0\right) ; \omega$ is the excitation frequency.

Within the inclusions, the base functions for the displacements field can be simply described by the full-space Green's functions [17], such that

$$
G_{x x}^{i}\left(x_{0}, y_{0}, x, y, \rho_{i}, \alpha_{i}, \beta_{i}\right)=G_{x x}^{f u l l}\left(x_{0}, y_{0}, x, y, \rho_{i}, \alpha_{i}, \beta_{i}\right)
$$

and 


$$
G_{y x}^{i}\left(x_{0}, y_{0}, x, y, \rho_{i}, \alpha_{i}, \beta_{i}\right)=G_{y x}^{f u l l}\left(x_{0}, y_{0}, x, y, \rho_{i}, \alpha_{i}, \beta_{i}\right),
$$

where $G_{i j}^{f u l l}(\cdot)$ represents the displacement generated along direction $i$, due to a load acting along $j$, by a dynamic source in an infinite elastic medium.

In the case of a half-space it is necessary to impose the following boundary conditions: null tangential and normal stresses at the half-space free surface. Thus, the systems of equations can be established for each value of $k_{n}$ that allow the unknown amplitude factors to be calculated. As an example, taking the specific case of a load acting along $x$, after solving the relevant equation systems for a full sequence of values of $k_{n}$, in the range $\left[-\frac{2 \pi}{L_{x}} N ;+\frac{2 \pi}{L_{x}} N\right]$, the final displacements contains an additional summation [17] due to the influence of the surface in order to satisfy the free-surface conditions:

$$
\begin{gathered}
G_{x x}^{h}\left(x_{0}, y_{0}, x, y, \rho_{h}, \alpha_{h}, \beta_{h}\right)= \\
G_{x x}^{f u l l}\left(x_{0}, y_{0}, x, y, \rho_{h}, \alpha_{h}, \beta_{h}\right)+E_{a} \sum_{n=-N}^{n=+N}\left(A_{n}^{x} \frac{-i k_{n}^{2}}{v_{n}} E_{b 0}-i \gamma_{n} E_{c 0} C_{n}^{x}\right) E_{d},
\end{gathered}
$$

and

$$
\begin{gathered}
G_{y x}^{h}\left(x_{0}, y_{0}, x, y, \rho_{h}, \alpha_{h}, \beta_{h}\right)= \\
G_{y x}^{f u l l}\left(x_{0}, y_{0}, x, y, \rho_{h}, \alpha_{h}, \beta_{h}\right)+E_{a} \sum_{n=-N}^{n=+N}\left(-i k_{n}^{2} A_{n}^{x} E_{b 0}+i k_{n} C_{n}^{x} E_{c 0}\right) E_{d} .
\end{gathered}
$$

\section{NUMERICAL IMPLEMENTATION AND VERIFICATION}

A comprehensive study was made in order to understand the accuracy and stability of the MFS in the analysis of different scenarios. A test case was analysed considering 6 solid circular inclusions with radius $R=0.5 \mathrm{~m}$. For the host medium, a shear modulus $G_{m}=43.52 \times 10^{6} \mathrm{~Pa}$ was considered. Both host medium and inclusion had a density $\rho_{m}=\rho_{i}=1700 \mathrm{~kg} / \mathrm{m}^{3}$ and Poisson's ratio $v_{m}=v_{i}=0.33$. The centre of the upper inclusions was placed at a depth of $2 \mathrm{~m}$ and spaced from each other $2 \mathrm{~m}$, either vertically or horizontally, as shown in Fig. 2.

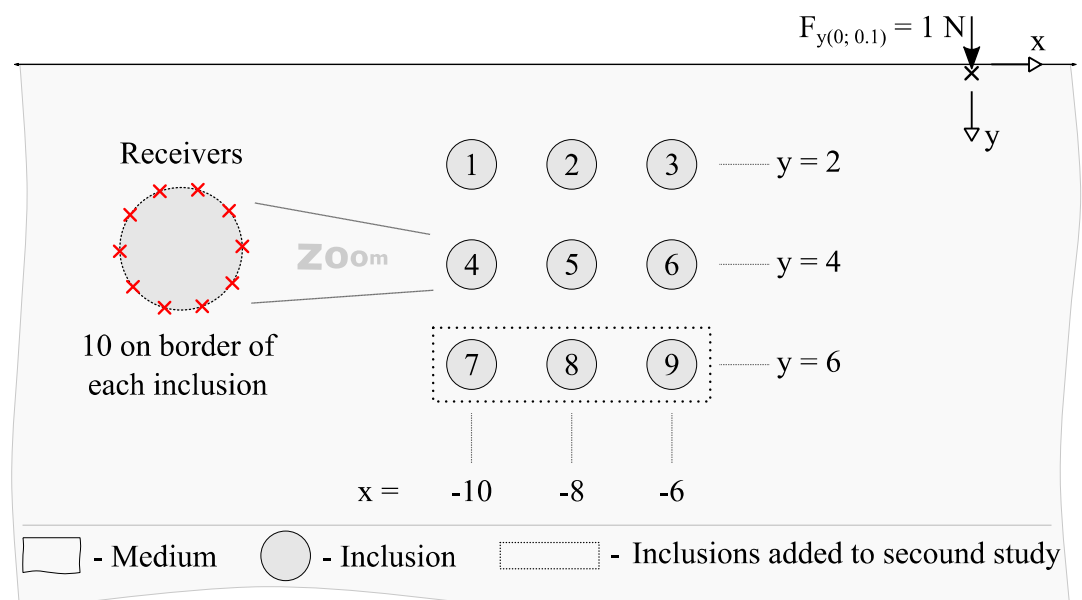

Figure 2: Schematic representation of multiple inclusion model. 
The response, in terms of horizontal $(x)$ and vertical $(y)$ displacements, is computed in $N E$ sets of 10 receivers evenly distributed along each inclusion border. Since receivers are placed over the interface between the inclusions and the host medium, the computation is performed by two possible ways: first using the result of eqn (5), as if receivers were in the host medium; and then the result of eqn (6), as if receivers were inside the inclusion.

Results were first computed for 6 inclusions with the same material properties of the host medium and excited by a frequency of $20 \mathrm{~Hz}$, with virtual sources positioned at $0.5 \mathrm{R}$ from the boundary. For this model (shown in Fig. 2) different collocation points were considered. For the case of 40 points, the imaginary (a) and real (b) parts of response are schematically represented in Fig. 3, where "•" markers are receivers on the original position; "०" markers represent the analytical response computed directly with the Green's functions (since the properties of the inner and outer materials are the same); " $x$ " markers represent the result computed as if the receivers were inside the inclusion using eqn (6); and "+" markers highlight results computed as if the receivers were in the host medium and calculated using the half-space Green's functions depicted in eqn (5). As can be seen in the figure, the numerical results match very well those calculated analytically, using the half-space Green's functions. The relative errors with respect to the analytical response are shown in Fig. 4 which presents the imaginary (a) and real (b) parts of relative errors for all different numbers of collocation points (between 20 and 80 ). Observing the presented plots, it can be concluded that the relative error tends to decrease with increasing numbers of collocation points, indicating a progressive convergence to the correct solution. Using 40 collocation points the relative error is already lower than $10^{-5}$, indicating excellent solution accuracy. Observing Fig. 4(c), it can be seen that the system matrix becomes progressively more ill-conditioned, although the quality of the calculations does not seem to be degraded due to this issue. It is indeed known that when using the MFS, and this parameter should be monitored to avoid numerical errors due to badly conditioned matrices.

An additional check was performed for different contrasts between the properties of the inclusion and those of the host medium. Results are shown in Fig. 5 through the representation of the deformed shape of the inclusion with the same stiffness ("○" markers), half stiffness (" $\times$ " markers) and twice the stiffness ("+" markers) of the host medium. This figure also compares these results with those achieved using the BEM under the same

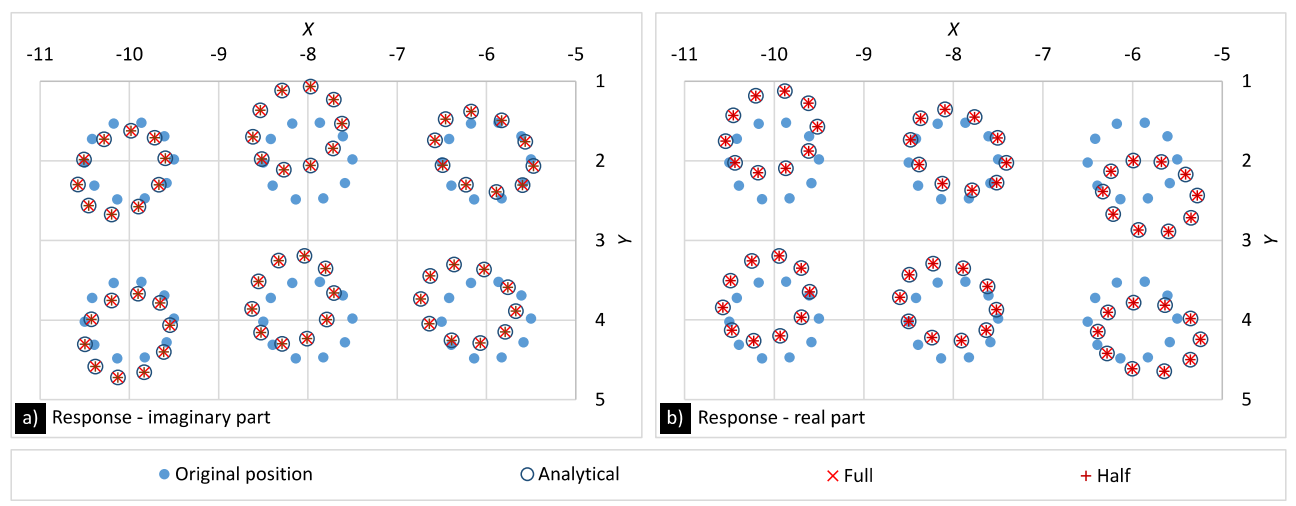

Figure 3: Deformed shape of the inclusions when 40 collocation points are used. (a) Imaginary; and (b) Real parts of the response, amplified by a factor of $1.5 \times 10^{8}$. 

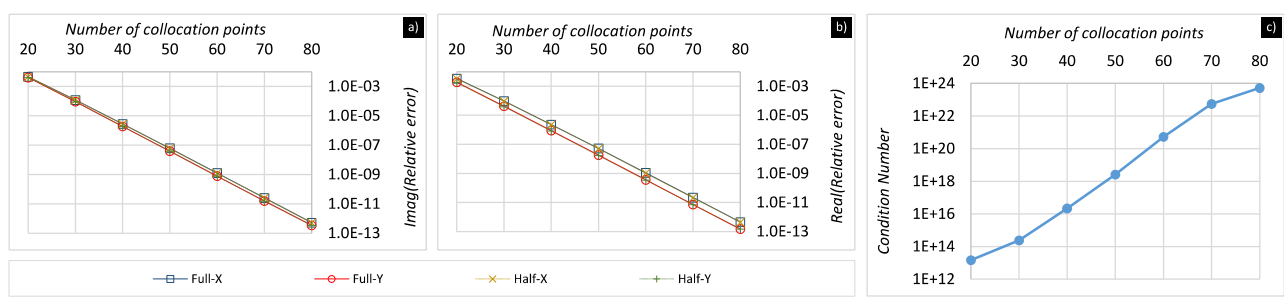

Figure 4: (a) Imaginary; (b) Real parts of relative errors; and (c) System matrix condition number.

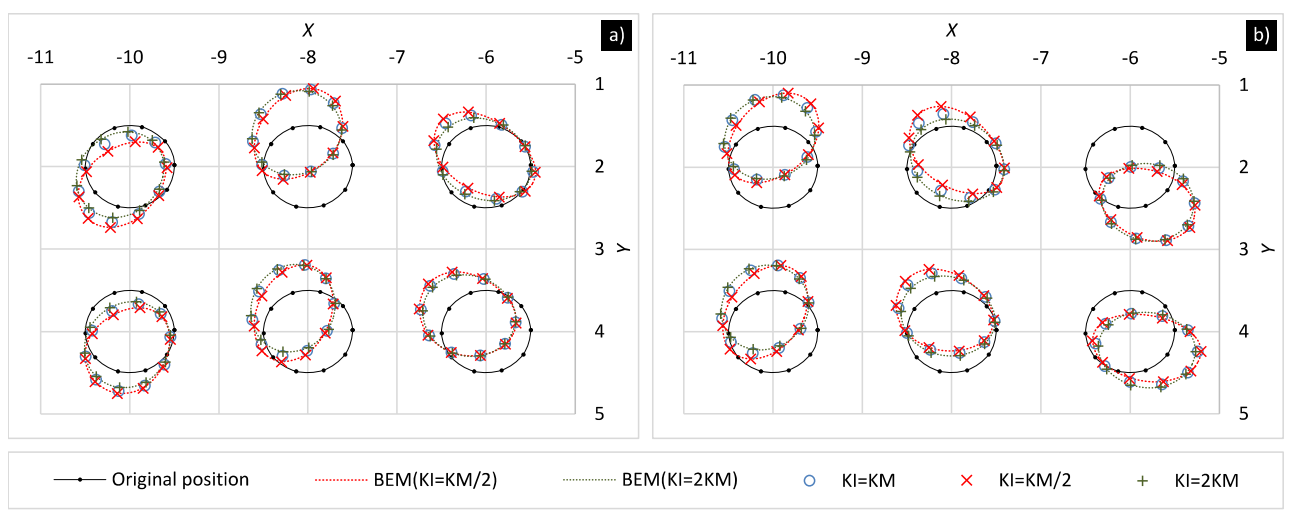

Figure 5: Modified positions of receivers. (a) Imaginary; and (b) Real parts of the responses, amplified by a factor of $1.5 \times 10^{8}$.

conditions. As can be seen, an excellent agreement of the MFS and BEM is obtained. Although not shown here, equally good results were obtained for larger sets of inclusions.

\section{NUMERICAL ANALYSIS}

To evaluate the effect of the presence of inclusions in the vibrations registered in receivers, it is evaluated a reduction in vibration levels. This reduction is computed in terms of an insertion loss, $I L$, that is defined as a ratio of the displacement vibration levels obtained in the presence of inclusions to the displacement vibration levels obtained without the inclusions. This ratio is given in $\mathrm{dB}$ by the following equation:

$$
I L_{i}=-20 \log \frac{\left|u_{i}\right|}{\left|G_{i j}\right|}
$$

where $u_{i}$ is the displacement field, along direction $i$, computed at receivers in presence of inclusions and $G_{i j}$ represents the displacement field, along direction $i$ and generated by a load acting along direction $j$, computed without the inclusions. According to eqn (13), positive values correspond to a reduction of the displacement vibration levels in the presence of inclusions and negative values of the insertion loss stand for losing protective solutions efficiency.

In this numerical study 4 different groups of solid circular inclusions will be compared, as shown in Fig. 6, buried in an elastic half-space at two depths (from the centre of the upper 
inclusion), (1 and 3) $\mathrm{m}$, and subjected to two frequencies, (15 and 120) $\mathrm{Hz}$ resulting from the dynamic vertical load $F_{y}=1 \mathrm{~N}$ acting near the surface at point $(0,0.1) \mathrm{m}$. All inclusions have a radius of $0.3 \mathrm{~m}$ and are made by a homogenous material with elastic properties of $\rho_{i}=2700 \mathrm{~kg} / \mathrm{m}^{3}, \quad G_{i}=1840 \times 10^{6} \mathrm{~Pa}$ and $v_{i}=0.20$, while the hosting soil is characterized by $\rho_{m}=1700 \mathrm{~kg} / \mathrm{m}^{3}, G_{m}=43.61 \times 10^{6} \mathrm{~Pa}$ and $v_{m}=0.33$. The inclusions are placed along the system axes equally spaced between them by a distance which is twice their diameter, and the centre of each group along horizontal direction is kept at $x_{G}=-15 \mathrm{~m}$.

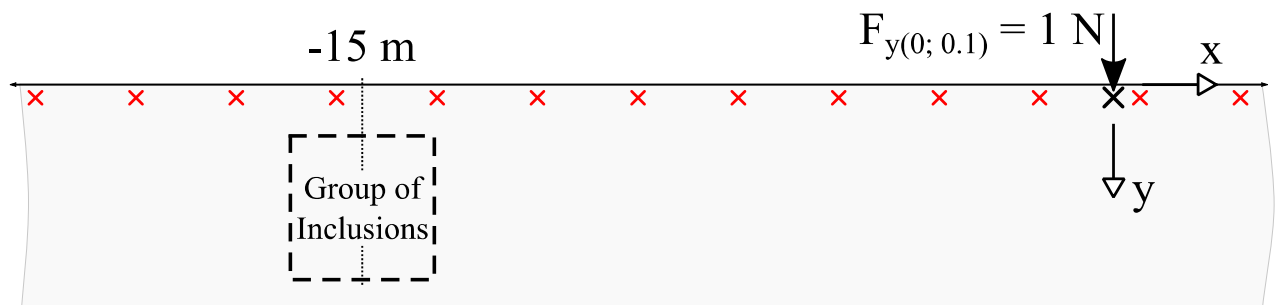

Group I.V Group I.H Group II.V Group II.H

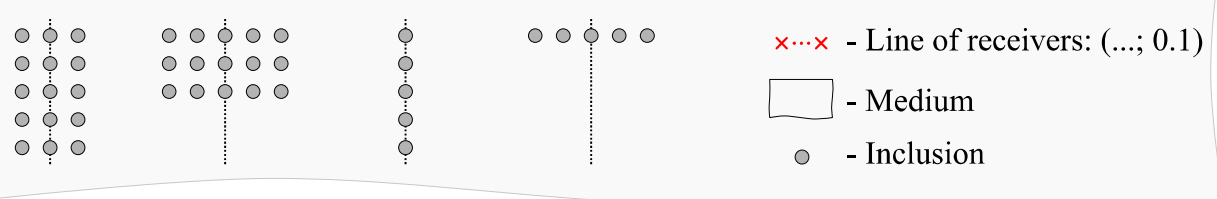

Figure 6: Model for the numerical application related to insertion loss.

All inclusions have a radius of $0.3 \mathrm{~m}$ and are made by a homogenous material with elastic properties of $\rho_{i}=2700 \mathrm{~kg} / \mathrm{m}^{3}, G_{i}=1840 \times 10^{6} \mathrm{~Pa}$ and $v_{i}=0.20$, while the hosting soil is characterized by $\rho_{m}=1700 \mathrm{~kg} / \mathrm{m}^{3}, G_{m}=43.61 \times 10^{6} \mathrm{~Pa}$ and $v_{m}=0.33$.

The response in terms of horizontal, $x$, and vertical, $y$, displacements is computed along a line of receivers placed $0.1 \mathrm{~m}$ below the surface and $0.1 \mathrm{~m}$ horizontally spaced in a range of $\{-30, \ldots, 30\} \mathrm{m}$. The computations are performed using the MFS model, with 30 collocation points distributed along the boundary of each inclusion, and with two sets of 30 virtual sources placed at either side of that interface, at a distance of $0.24 \mathrm{~m}$. Fig. 7 illustrates the insertion loss, in $\mathrm{dB}$, along the line of receivers for $x$ and $y$ components of the displacements and shows the influence in the response of the depth ( $1 \mathrm{~m}$ or $3 \mathrm{~m})$ of inclusions Group I.V (see Fig. 6) and Group I.H, and for frequencies of $15 \mathrm{~Hz}$ and $120 \mathrm{~Hz}$. Considering Group I.V (Fig. 7(a)), it can be concluded that for low frequencies $(15 \mathrm{~Hz})$ the influence of the depth of the inclusions is not very perceptible, and low attenuation values are always registered. However, at a higher frequency $(120 \mathrm{~Hz})$ the effect of this geometric parameter reveals to be determinant in the effect provided by the presence of the inclusions. At receivers placed between the inclusions zone and the sensible structures (downstream zone), considerable attenuation values (positive $I L$ values) are identified, for the lowest depth values. Considering now Group I.H (rotating the inclusions Group I.V by $90^{\circ}$ ), the computed results at the same line of receivers are presented in Fig. 7(b). Again, it is clear that for the lower frequency, with larger wavelengths, very small attenuations are registered, while a much stronger attenuation effect is seen in the downstream zone for the higher frequency, and for inclusions closer to the surface. Comparing the results computed for both cases, it 
can be seen that a stronger attenuation is observed when a larger number of inclusions is distributed along the horizontal direction, thus allowing a stronger interference with waves traveling closer to the surface.

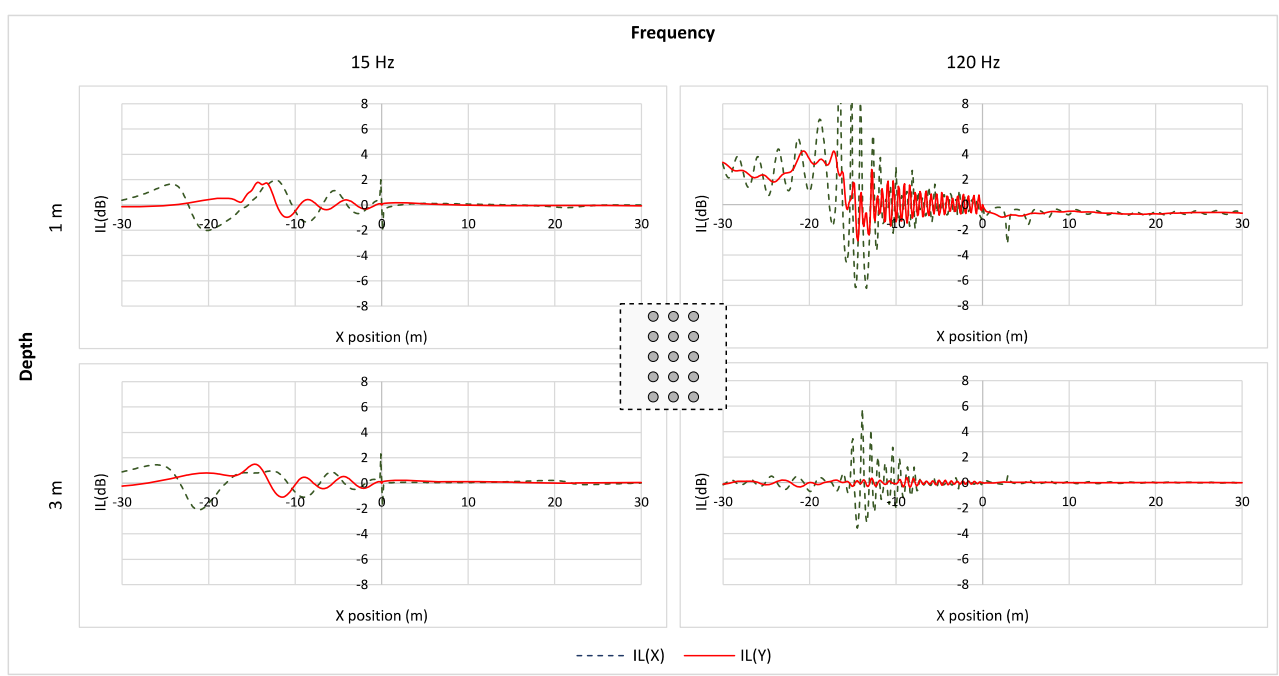

(a)

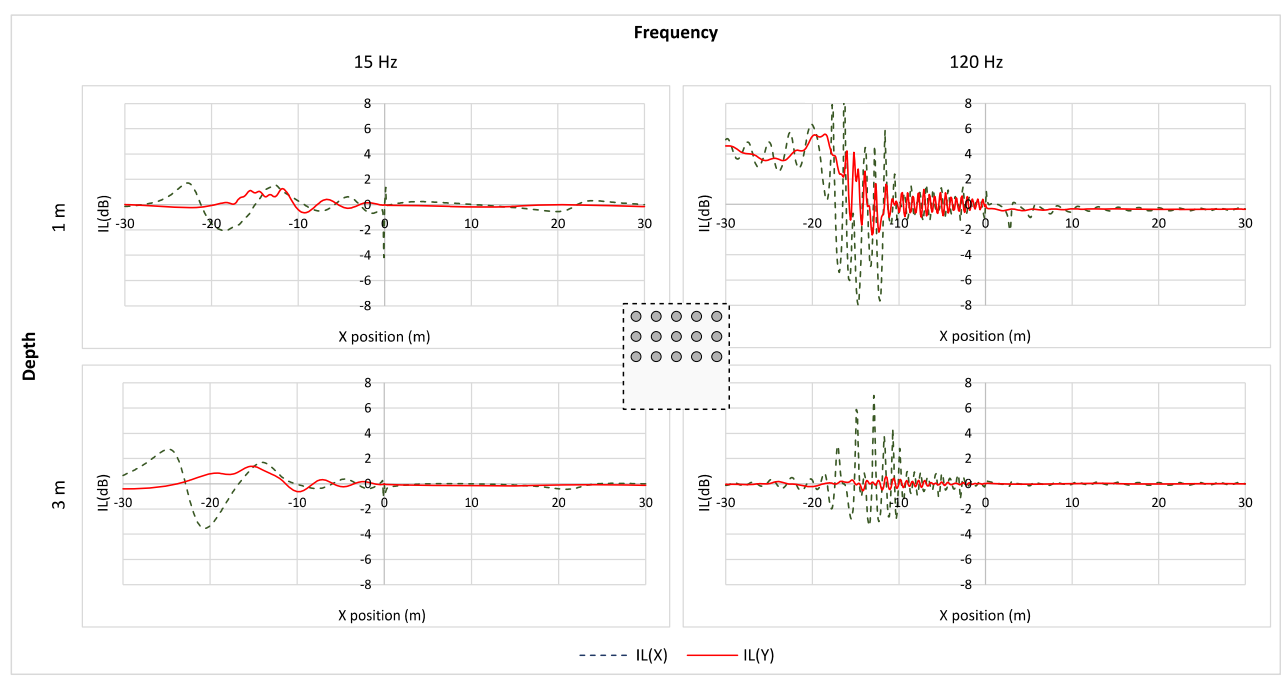

(b)

Figure 7: Attenuation provided by (a) Group I.V; and (b) Group I.H.

In order to identify the effect of varying number of inclusions, relative to the previous model, consider now just the presence of one line (Group II.H) with 5 inclusions, whose results can be seen in Fig. 8. Analysing this set of results, it becomes clear that, for the higher frequency, the effect provided by just one line of inclusions is very similar to that obtained when three horizontal lines are included. When a lower frequency is considered, the larger 
wavelengths involved lead again to a smaller attenuation when only one line of inclusions is used.

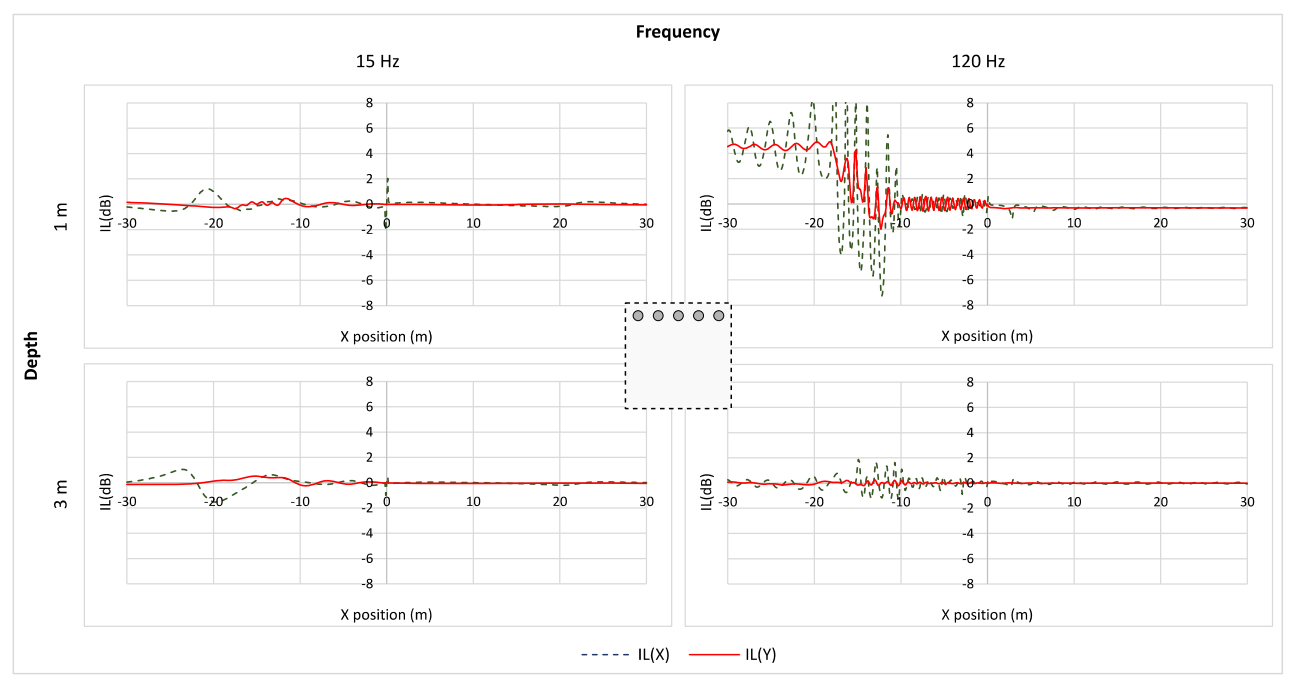

Figure 8: Attenuation provided by Group II.H.

To better asses the attenuation provided by the different configurations, Fig. 9 illustrates the insertion loss (in $\mathrm{dB}$ ) for each of the inclusions' groups in one third octave bands and computed in the receivers placed on the downstream zone. Clearly, when only one vertical line of inclusions is considered very low insertion loss values are registered throughout the frequency range analysed. When more inclusions are considered along the horizontal direction, again very low attenuations are registered at lower frequencies, but starting on the $63 \mathrm{~Hz}$ band a much stronger insertion loss is visible. Indeed, considering the propagation velocities allowed by the host medium (around $247 \mathrm{~m} / \mathrm{s}$ for P waves, $160 \mathrm{~m} / \mathrm{s}$ for S waves and $150 \mathrm{~m} / \mathrm{s}$ for Rayleigh waves), this frequency band coincides with the so-called "phononic crystal effect", generated by the Bragg interference phenomenon occurring between inclusions, which occurs approximately around $\mathrm{c} / 2 \mathrm{~d}$ (c being the velocity and $\mathrm{d}$ the spacing between inclusions). As more inclusions are considered along the horizontal direction, this effect becomes stronger, and leads to stronger attenuations, as is clear in the provided results. This results confirms that this type of mitigation strategy may be designed to fit a given frequency of the propagating vibration field, thus originating a good attenuation and providing effective protection for sensible structures positioned the beyond the inclusions.

\section{CONCLUSIONS}

In this work, a general MFS strategy was presented to allow the analysis of wave propagation in the presence of buried inclusions in a half-space. The proposed strategy is general and allows the simulation of any number of inclusions filled with distinct materials. The presented numerical results evidence the stability and convergence of solutions. The accuracy of the MFS is quite good, particularly when compared with BEM results. This paper only reported findings concerning the 2D case, and further in-depth studies need to be conducted to verify and improve the proposed strategy, in particular for $2.5 \mathrm{D}$ and $3 \mathrm{D}$ cases. Conceptually, the 


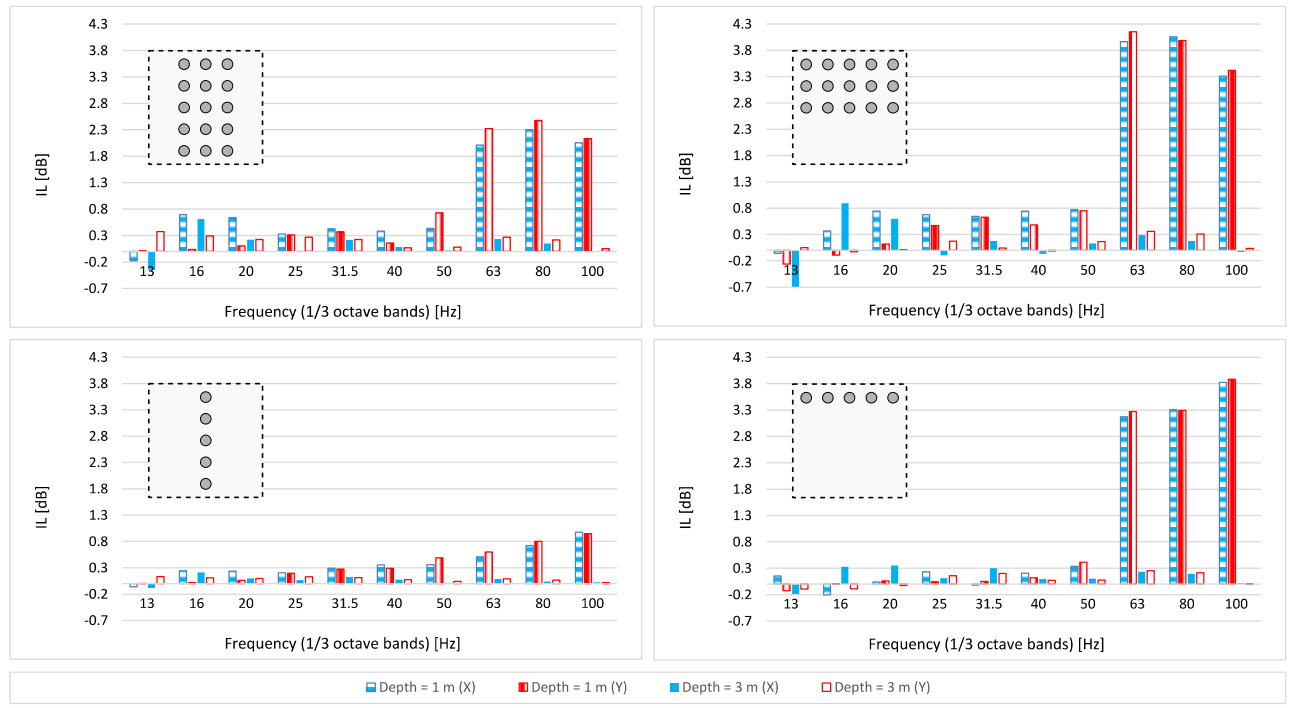

Figure 9: Insertion loss in 1/3 octave bands.

inclusions have a significant effect on vibration levels reduction, and it was observed that their efficiency peaks at a specific frequency range, which is strongly related to the Bragg effect occurring due to the multiple interferences between reflections at the equally spaced structures.

\section{ACKNOWLEDGEMENTS}

This work was financially supported by: Project PTDC/ECM-COM/1364/2014 - POCI-010145-FEDER-016783 (METASHIELD) - funded by FEDER funds through COMPETE2020 - Programa Operacional Competitividade e Internacionalização (POCI) and by national funds through FCT - Fundação para a Ciência e a Tecnologia, I.P. This work was partly financed by FEDER funds through the Competitivity Factors Operational Programme COMPETE and by national funds through FCT - Foundation for Science and Technology within the scope of the project POCI-01-0145-FEDER-007633 (ISISE) and through the Regional Operational Programme CENTRO2020 within the scope of the project CENTRO01-0145-FEDER-000006. D. Dias-da-Costa would like to acknowledge the support from the Australian Research Council through its Discovery Early Career Researcher Award (DE150101703) and ARC Projects (DP140100529, LP140100591).

\section{REFERENCES}

[1] Yang, Y.B. \& Hung, H.H., Wave Propagation for Train-Induced Vibrations. A Finite/Infinite Element Approach, World Scientific Publishing: Singapore, 2009.

[2] Al Suhairy, S., Prediction of Ground Vibration From Railways, SP Swedish National Testing and Research Institute: Sweden, 2000.

[3] Croy, I., Smith, M.G. \& Waye, K.P., Effects of train noise and vibration on human heart rate during sleep: An experimental study. BMJ Open, 2013.

DOI: 10.1136/bmjopen-2013-002655. 
[4] Lombaert, G., Degrande, G., Vanhauwere, B., Vandeborght, B. \& François, S., The control of ground-borne vibrations from railway traffic by means of continuous floating slabs. Journal of Sound and Vibration, 297(3-5), pp. 946-961, 2006.

[5] Andersen, L., Frigaard, P. \& Augustesen, A., Mitigation of ground vibration by double sheet-pile walls. Geotechnical Engineering/Construction Technology and Management: Proceedings of the 8th International Congress on Advances in Civil Engineering, $A C E$ 2008, Famagusta, North Cyprus, 2008.

[6] Ang, W.T., A Beginner's Course in Boundary Element Methods, Universal Publishers: Boca Raton, USA, 2007.

[7] Brebbia, C.A. \& Dominguez, J., Boundary element methods for potential problems. Applied Mathematical Modelling, 1(7), pp. 372-378, 1977.

[8] Tsiatas, G.C.\& Yiotis, A.J., A BEM-based meshless solution to buckling and vibration problems of orthotropic plates. Engineering Analysis with Boundary Elements, 37(3), pp. 579-584, 2013.

[9] Godinho, L., Amado-Mendes, P. \& Tadeu, A., Meshless analysis of soil-structure interaction using an MFS-MLPG coupled approach. Engineering Analysis with Boundary Elements, 55, pp. 80-92, 2015.

[10] Fries, T.-P. \& Matthies, H.-G., Classification and Overview of Meshfree Methods, Institute of Scientific Computing, Technical University Braunschweig: Brunswick, Germany, 2004.

[11] Godinho, L.M.C., Costa, E.G.A., Pereira, A.S.C. \& Santiago, J.A.F., Some observations on the behavior of the method of fundamental solutions in 3D acoustic problems. International Journal of Computational Methods, 9(04), 1250049, 2012.

[12] Tadeu, A., António, J. \& Godinho, L., Defining an accurate MFS solution for 2.5D acoustic and elastic wave propagation. Engineering Analysis with Boundary Elements, 33(12), pp. 1383-1395, 2009.

[13] Gu, Y., Chen, W. \& Zhang, C.-Z., Singular boundary method for solving plane strain elastostatic problems. International Journal of Solids and Structures, 48(18), pp. 2549-2556, 2011.

[14] Soares, Jr. D. \& Godinho, L., An overview of recent advances in the iterative analysis of coupled models for wave propagation. Journal of Applied Mathematics, 426283 2014.

[15] Godinho, L., Amado-Mendes, P., Pereira, A. \& Soares, Jr. D., A coupled MFS-FEM model for 2-D dynamic soil-structure interaction in the frequency domain. Computers and Structures, 129, pp. 74-85, 2013.

[16] Tadeu, A., António, J. \& Godinho, L., Green's function for two-and-a-half dimensional elastodynamic problems in a half-space. Computational Mechanics, 27, pp. 484-491, 2001.

[17] Tadeu, A. \& Kausel, E., Green's functions for two-and-a-half dimensional elastodynamic problems. Journal of Engineering Mechanics, 126(10), pp. 1093-1097, 2000 . 